COMMENT

\title{
MRI at term equivalent in preterm infants: the wise choice
}

\author{
Donna M. Ferriero' \\ Pediatric Research (2018) 84:791-792; https://doi.org/10.1038/s41390-018-0068-y
}

In the article in this issue titled: "Brain imaging in preterm infants $<32$ weeks gestation: a clinical review and algorithm for the use of cranial ultrasound and qualitative brain MRI", ${ }^{1}$ strong scientific argument is given to answer this question in the affirmative. The authors have addressed the complex physiology of preterm brain injury and analyze studies using both cranial ultrasound and magnetic resonance imaging (MRI) that show how this physiology contributes to the injury. The sensitivity of the two modalities is contrasted for white matter injury (MRI) and cerebellar pathology, including hemorrhage.

It is clear from the data that cranial ultrasound is adequate for evaluating intraventricular hemorrhage and post-hemorrhagic hydrocephalus, but it is woefully inadequate in delineating the extent of white matter injury especially when it is diffuse. Cranial ultrasound also falls short in evaluating cerebellar pathology and hemorrhages because of the limitations of the mastoid view. The investigators cite four studies outlined in Table 1 that make the comparison of cranial ultrasound with MRI. Each study showed that cranial ultrasound was inferior to MRI in detecting diffuse white matter injury (WMI), small petechial hemorrhages, especially in the posterior fossa, and cerebellar hypoplasia due to limitations of the mastoid views. Since most of the white matter injury over the last decade is no longer cystic, the limitations in detecting non-cystic white matter changes become even more apparent. ${ }^{2}$

The ability of MRI to predict neurodevelopmental outcome is also superior to that of cranial ultrasound. Ibrahim et al. ${ }^{1}$ outline 11 studies in their analysis. All studies concluded that MRI is superior. One of the largest prospective trials to date, the NEURO study, ${ }^{3}$ showed that the increasing severity of white matter injury assessed by term-equivalent MRI was significantly associated with lower mean BSID II cognitive scores, and moderate-to-severe Cerebral Palsy (CP). These findings substantiated the reports by many centers. In addition, a systematic review and meta-analysis, the predictive value of MRI at term-equivalent age for neurodevelopmental outcomes showed that pooled sensitivity and specificity values for prediction of $\mathrm{CP}$ were $77 \%$ and $79 \%$, respectively. ${ }^{4}$

Recently it has been shown that the cerebellum is affected by the encephalopathy of prematurity, and commonly exhibits both hemorrhages and hypoplasia. Again, the superiority of MRI over cranial ultrasound was shown in multiple studies. The importance of identifying cerebellar lesions lies in the fact that these abnormalities correlate with poorer IQ and motor outcomes. Studies of premature cerebella using high-field-strength MRI have led to the discovery of an unexpectedly high incidence of cerebellar hemorrhage in prematurely born neonates..$^{5}$ These hemorrhages are associated with localized cerebellar hypoplasia. Data suggest that affected patients have learning and behavioral disabilities, in addition to motor dysfunction. Of some comfort is that, in a study to be released this month from Utrecht, ${ }^{6}$ small punctate cerebellar lesions did not affect supratentorial white matter development or subsequent cognitive or overall motor function in late infancy. Further follow-up needs to be done to assess the cognitive and motor outcomes at school age.

Newer data with more sophisticated MR analyses provide even more important information. Two recent studies illustrate this point.

In a recent study from the Miller group, Guo et al. ${ }^{7}$ show that WMI occurs in a characteristic topology, with most lesions occurring in a periventricular pattern, followed by lesions occurring first in posterior white matter and then in frontal regions. In this study, probabilistic maps demonstrated that location of the lesion adds important prognostic information for predicting motor, cognitive and language, and that the frontal lesions were of particular concern for poor outcome. The WMI volume increases seen in the frontal regions were key predictors of adverse cognitive and motor outcomes. These results improve our ability to identify those neonates who need early interventions and therapeutic strategies to enhance brain repair.

Another prospective population-based cohort study from Sweden published early this year investigated the relationship between neonatal brain volumes and visual motor integration and fine motor skills in preterm born children at 6.5 years of age. ${ }^{8}$ These children had neither major brain lesions nor cerebral palsy, yet when the term-equivalent scans were analyzed with automated segmentation techniques, the volume of the precentral gyrus showed a positive correlation with both visual-motor integration and fine motor skills. In addition, the volumes of the cerebellum and the brainstem correlated positively with fine motor skills. Again, their conclusions were similar to those of the Miller group that the clear associations between early brain volume alterations and later visual-motor integration and/or fine motor skills make early interventions possible.

As part of the "Choosing Wisely Campaign", 9 the routine screening of term-equivalent or discharge brain MRIs in preterm infants was suggested to be "overtreatment". They defined overtreatment as "waste that comes from subjecting patients to care that, according to sound science and the patient's own preferences, cannot help them". One must then ask whether prognostic information is beneficial to families. As reviewed by Ibrahim et al., a randomized trial conducted in the United Kingdom assessing term-equivalent MRI and counselling was shown to reduce parental anxiety. The impact of parents' mental health on the health and well-being of their newborn is increasingly appreciated. A recent study showed that mothers of infants born preterm were more likely to report mental health disorders and perceive themselves as less ready to go home with their fragile newborns. ${ }^{10}$ Although MRI scanning in this project

\footnotetext{
${ }^{1}$ Weill Institute for Neurosciences, Newborn Brain Research Institute, University of California San Francisco, 675 Nelson Rising Lane Rm415, San Francisco, CA 94143-0663, USA Correspondence: Donna M. Ferriero (Donna.ferriero@ucsf.edu)
}

Received: 9 April 2018 Revised: 2 May 2018 Accepted: 11 May 2018 Published online: 28 June 2018 
was associated with a minor increase in health-related costs, I suggest that this cost is justified to help the patient's family. It is trivial compared to a NICU hospitalization. Should this incremental cost then be the sole driver of our recommendations?

The "Choosing Wisely" publication concluded that there is insufficient evidence that routine use of MRI in preterm infants improves long-term outcome. Unfortunately, the authors are confusing prediction and outcome. No one is claiming that a scan can improve outcome, but rather that performing the scan allows one to decide on necessary interventions that might improve outcome. Such a study has yet to be done, and stratification may be essential. In a letter to the editor in response to the study by Anderson, ${ }^{10}$ the authors of the "Choosing Wisely" statement say that "Studies must show that term-equivalent brain MRIs are superior to neurodevelopmental evaluations, or less expensive than more accessible technologies like intracranial ultrasound, to warrant routine use". This is indeed what is in the published literature. Thus, a study needs to be done to test whether interventions based on MRI results at term-equivalent age can improve outcome, not that the studies themselves improve outcomes. Currently, the scan is done to help the family plan and prepare for necessary care and treatment if abnormalities are detected on the term-equivalent MRI. Furthermore, in providing important prognostic information, MRI provides clinicians with an opportunity to address parental anxiety and to reassure the family if normal. Many studies, as outlined in Ibrahim et al. ${ }^{1}$ and in the literature, show that an MRI done at this time correlates with neurodevelopmental outcomes at 2 and 5 years of age.,11 The improved neurodevelopmental outcomes in children who have abnormal term-equivalent age brain MRIs will be confounded by the severity of injury on that scan. Therapeutic interventions may improve those outcomes, but it it is likely to be incremental in the most severe cases. However, the information that the parents receive will be critical in their decision-making such as schooling for their child. Engaging parents in these discussions early on should allow their needs as a family to come first.

Ibrahim et al. ${ }^{1}$ propose an algorithm for imaging asymptomatic preterm neonates $<32$ weeks gestation. They suggest that for the 29-32 weeks or $<1500 \mathrm{~g}$ baby only ultrasound should be performed. They propose a term-equivalent MRI for preterm neonates born $<29$ weeks or $<1000 \mathrm{~g}$, if it can be done without sedation. Obviously any preterm newborn who is symptomatic deserves an MRI for clinical indications at any point, especially for acute events like hypoxic-ischemic encephalopathy, seizures, infection, or stroke.

I strongly disagree, and recommend that even asymptomatic 29-32-week gestation newborns should have an MRI at term- equivalent age. These neonates are just as likely to suffer from the encephalopathy of prematurity, and have WMI and cerebellar hypoplasia. Therefore, despite the well intentioned guidelines provided by the "Choosing Wisely" campaign, the preterm newborn who is at high risk of brain injury should have proper documentation of their brain status by MRI prior to discharge from the neonatal intensive care unit.

\section{ACKNOWLEDGEMENTS}

I thank Steven P. Miller for thoughtful review of the commentary. D.M.F. was funded by P01NS082330 (NINDS/NIH).

\section{ADDITIONAL INFORMATION}

Competing interests: The authors declare no competing interests.

Publisher's note: Springer Nature remains neutral with regard to jurisdictional claims in published maps and institutional affiliations.

\section{REFERENCES}

1. Ibrahim, J., Mir, I. \& Chalak, L. Brain imaging in preterm infants $<32$ weeks gestation: a clinical review and algorithm for the use of cranial ultrasound and qualitative brain MRI. Pediatr Res. (in press).

2. Gano, D. et al. Diminished white matter injury over time in a cohort of premature newborns. J. Pediatr. 166, 39-43 (2015).

3. Hintz, S. R. et al. Neuroimaging and neurodevelopmental outcome in extremely preterm infants. Pediatrics 135, e32-e42 (2015).

4. Van't Hooft, J. et al. Predicting developmental outcomes in premature infants by term equivalent MRI: systematic review and meta-analysis. Syst. Rev. 4, 71 (2015).

5. Kim, $\mathrm{H}$. et al. Hindbrain regional growth in preterm newborns and its impairment in relation to brain injury. Hum. Brain. Mapp. 37, 678-688 (2015).

6. Senden, R. E. M. et al. Mild cerebellar injury does not significantly affect cerebral white matter microstructural organization and neurodevelopmental outcome in a contemporary cohort of preterm infants. Pediatr. Res. 83, 1004-1010 (2018).

7. Guo, T. et al. Quantitative assessment of white matter injury in preterm neonates: association with outcomes. Neurology 88, 614-622 (2017).

8. Bolk, J. et al. Visual-motor integration and fine motor skills at $6(1 / 2)$ years of age and associations with neonatal brain volumes in children born extremely preterm in Sweden: a population-based cohort study. BMJ Open 8, e020478 (2018).

9. Ho, T. et al. Choosing wisely in newborn medicine: five opportunities to increase value. Pediatrics 136, e482-e489 (2015).

10. McGowan, E. C. et al. Maternal mental health and neonatal intensive care unit discharge readiness in mothers of preterm infants. J. Pediatr. 184, 68-74 (2017).

11. Setanen, S., Haataja, L., Parkkola, R., Lind, A. \& Lehtonen, L. Predictive value of neonatal brain MRI on the neurodevelopmental outcome of preterm infants by 5 years of age. Acta Paediatr. 102, 492-497 (2013). 